\title{
THE ORIGIN OF ABNORMAL RETINAL CORRESPONDENCE
}

\author{
BY
}

\author{
T. A'B. Travers \\ MELBOURNE
}

THIs essay is the final report of a British Medical Association research scholarship. The early portion of the investigation was described in a previous paper (1).

The ideas set out here follow naturally upon the first report, and it is essential that the latter be understood for the ideas to be readily comprehended. For this reason, I will briefly recapitulate the findings which are particularly relevant to the present thesis.

In concomitant squint there are two main types of suppression occurring in the deviated eye :

1. Suppression of the false image of the object of regard. This image, if not suppressed, would cause diplopia. The suppression is easy because the image upon the fixing macula is so much clearer than that upon the peripheral retina of the deviated eye.

This type of suppression does not vary with retinal correspondence. The typical suppression scotoma may be demonstrated by a method which I described as the "Colour Test" (1).

2. Suppression of the area of retina in the squinting eye which has the same "local sign " $\dagger$ as the fixing macula. This suppression prevents a " confusion."*

Suppression of this variety is intimately associated with retinal correspondence. The typical suppression scotomata may be easily demonstrated by a method I have called "The mirror-screen test" (1).

If correspondence is normal, there is only very slight macular suppression in the deviated eye-unless there is amblyopia also.

If retinal correspondence is abnormal, there is a much larger and deeper area of suppression in an eccentric part of the retina of the deviated eye.

Now, abnormal retinal correspondence is an abnormality of binocular vision and is a binocular phenomenon.

So, although I have referred above to suppression scotomata

† The " Local Sign "3 of a sensory impression is the inherent quality that conveys to the brain the place of origin of the impulse. Thus, if we are touched on the hand, the local sign of the sensory impression is referred by the brain to the part of the hand touched. Retinal local signs inform us of the direction of objects. The local sign of the macula is the "straight ahead" direction.

*A "Confusion" is the name given by Von Kries ${ }^{2}$ to the phenomenon of an image from the squinting eye which appears in consciousness as if it were superimposed upon the fixation object. This is different from a diplopia image which is seen to one side of the fixation object. 
that occur " in the deviated eye," one should think of each eye and monocular field as an integral portion of the binocular field. It is convenient to refer to a suppression scotoma that occurs in one eye-but one should not forget that this suppressed area is really in a part of the binocular field.

Suppression can only exist in one eye if the other eye is fixing. One part of the binocular field can only be suppressed so long as fixation is present.

Much of the difficulty of understanding abnormal correspondence arises in this manner. The field of each separate eye in a squinter is normal. It is only when the eyes are examined binocularly that an abnormality is present. For-just as a squint depends upon the relative positions of the two eyes (for one eye cannot squint by itself) so the visual abnormalities of squint depend upon the functioning together of both eyes.

In the normal person, then, the two monocular fields overlap with little or no macular suppression. This is because the two visual lines intersect upon the object of fixation, and this allows the two maculae to be presented with similar images. A fluctuating and alternating slight macular suppression is simply a manifestation of normal retinal rivalry. If the macula of one eye habitually holds the ascendency in binocular vision, then that eye may be called the " master-eye."

Even in the normal person, there is suppression of the physiological diplopia images in the peripheral field.

In squint the visual lines are not both directed upon the fixation object, and thus suppression arises in the binocular field.

The diplopia image is suppressed irrespective of the type of retinal correspondence.

A confusion is prevented by suppression of that part of the binocular field which has the same local sign as the fixing macula. The position of this area will vary according to the correspondence.

Thus, if correspondence is normal, the part of the field that is suppressed will be in a line with the macula of the deviated eye. If correspondence is abnormal, the portion suppressed will be in line with an eccentric part of the retina.

I have shown that the suppression scotomata associated with abnormal correspondence alter as the retinal correspondence changes. This is typically seen after a corrective operation. The size of the scotoma is roughly proportional to the angle of anomaly. As the latter diminishes, so does the area of suppression. When retinal correspondence becomes normal again, suppression ceases.

Now can one argue, in the reverse way, that macular suppression is the first stage in the development of abnormal correspondence? 
This problem is associated with the interpretation of direction.

The relative position of objects is determined by their relation to the visual line. Thus one says that a thing is to the "right" or "left " of some fixation object. The visual line is taken as the central line around which all directions are orientated.

The power of visually discriminating direction then, must depend largely upon a single spot of high visual acuity (which

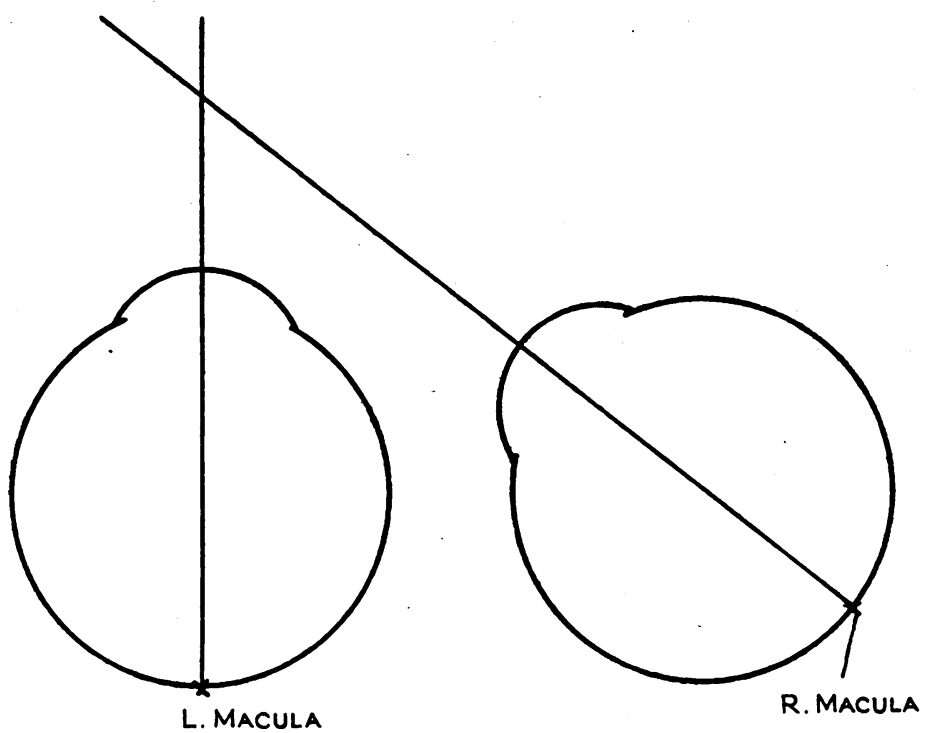

FIG. 1.

forms the central spot of fixation) surrounded by a large area of low visual acuity.

Impressions which arise in the "low acuity areas" of the retina are then easily orientated in relation to the central visual line.

In the squinter there is an alteration in the binocular field because there are two visual lines pointing in different directionsas in Fig. 1. Let us suppose that Fig. 1 represents the eyes of a child who has only recently commenced to squint. His retinal correspondence will be normal.

If suppression of some part of the binocular field did not occur, then there would ensue a confusion. The object imaged upon the macula of the deviated eye would " rise up into consciousness" as if it were superimposed upon the fixation object. This is prevented by a macular suppression in the deviated eye-or more accurately, by a suppression of a part of the binocular field in line with the deviated macula.

As this occurs, the visual acuity curve of the deviated eye is greatly altered as in Fig. 2. 


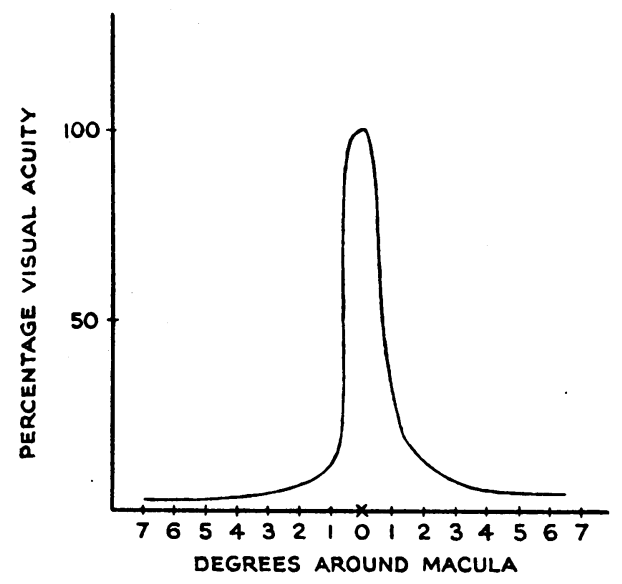

(A) NORMAL

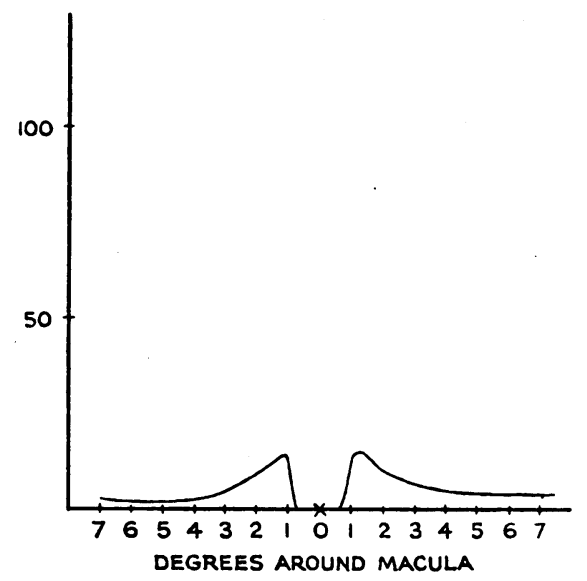

(B) DURING FIXATION OF OTHER EYE

FIG. 2

It follows, then, that while one eye is fixing, the power of the other for discriminating direction will be greatly altered.

There will still be only one visual line in the binocular fieldthat of the fixing eye. Directions of objects will be orientated around this central point. The macular suppression in the

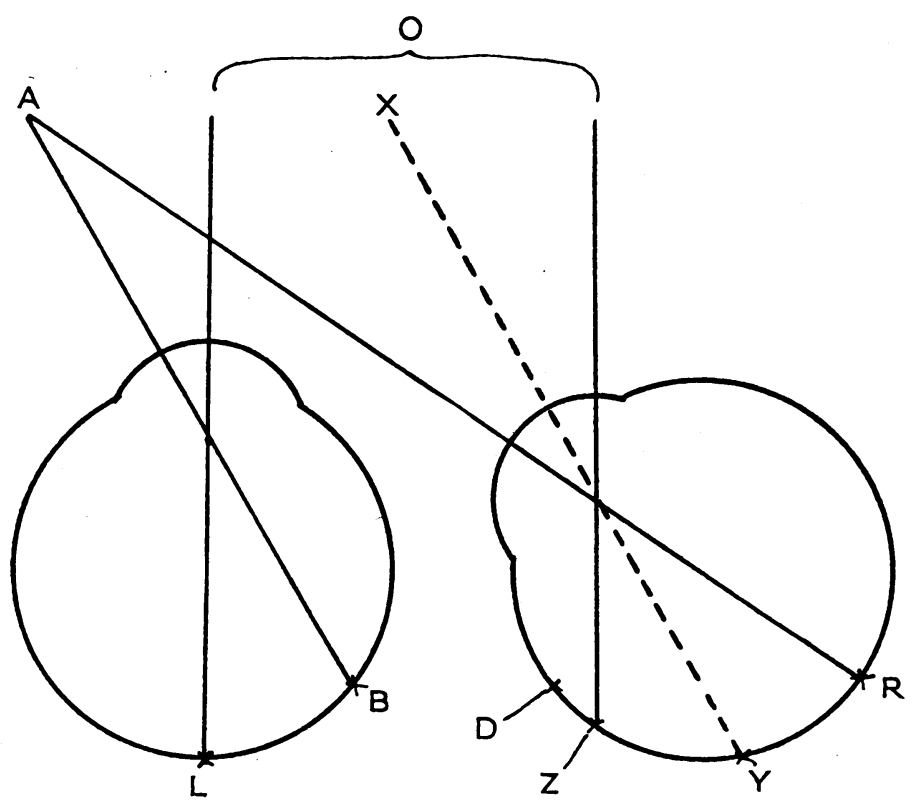

FIG. 3 . 
deviated eye will prevent any disturbing influences, and it will alter the normal power of the deviated eye or part of the field of discriminating direction.

Now suppose that Fig. 3 represents a squinter, with normal correspondence, fixing a distant object $O$ with the left eye. The image of $\mathrm{O}$ at $\mathrm{Z}$ will be suppressed (to avoid diplopia). Because of the suppression of $R$ (to avoid confusion) the only image of $A$ to reach consciousness will be that in the fixing eye at $B$.

Therefore, at the moment when L. fixes, the position of $A$ will be interpreted as being to the left of $\mathrm{O}$-and not as if it were superimposed on $\mathrm{O}$ (as it should be, because $\mathrm{L}$. and $\mathrm{R}$. have the same local signs or are corresponding points).

Thus when L. fixes, the meridian AR will gradually be interpreted by the right or deviated part of the field as having the directional value of a position to the left of the fixation point. Then another meridian-say XY-will assume by juxta position to $A R$ the same directional value as the visual line of the fixing eye. This is an abnormal correspondence.

Then $Y$ will have the same local sign as the fixing macula $L$, and $\mathrm{Y}$ will be suppressed in turn-to prevent a confusion. (The area of suppression around $\mathrm{Y}$ will be much larger than that around $R$, because the visual acuity of $Y$ is much lower than that of R.)

This shows how the angle of anomaly increases. The point $Y$ would move away from $\mathrm{R}$, constantly preventing confusion.

This would continue until the suppression scotoma around $\mathrm{Y}$ included the point $\mathrm{Z}$ - which lies in the same visual direction as the point $O$. When this occurs there would be no further tendency to increase, as any point to the left of $\mathrm{Z}$ (say D) has, in both eyes, a directional value which is to the right of the fixation point.

This theory is well substantiated by experimental evidence.

As regards the first stage in the development of an abnormal correspondence, it is very hard to get absolute proof because the subjects are necessarily very young. However, I have seen two children who undoubtedly had normal retinal correspondence when first tested by the synoptophore. Their squints gradually became worse and they developed an abnormal correspondence. I was unable to test them with the mirror-screen as their powers of concentration were insufficient. However, in each case it was clear that they developed first a macular suppression and then the abnormal correspondence. I was unable to show any intermediate stage as the angle of anomaly altered.

It will also be seen in mirror-screen tests that an angle of anomaly of much over $15^{\circ}$ is uncommon. This is because the suppression is so great that (in Fig. 3) the scotoma around Y has 
reached $\mathrm{Z}$, and there is no further tendency for confusion, as described above.

The reverse process-the way in which the abnormal correspondence changes after operation and gradually becomes normal, may be seen clinically. The gradual lessening of suppression as the angle of anomaly changes is easily demonstrated on the mirror-screen.

The explanation of how this happens is given below. (It is the exact reverse of the process described earlier.)

In Fig. 4 the right eye was formerly convergent, but the eyes are now straight after operation. There used to be an abnormal

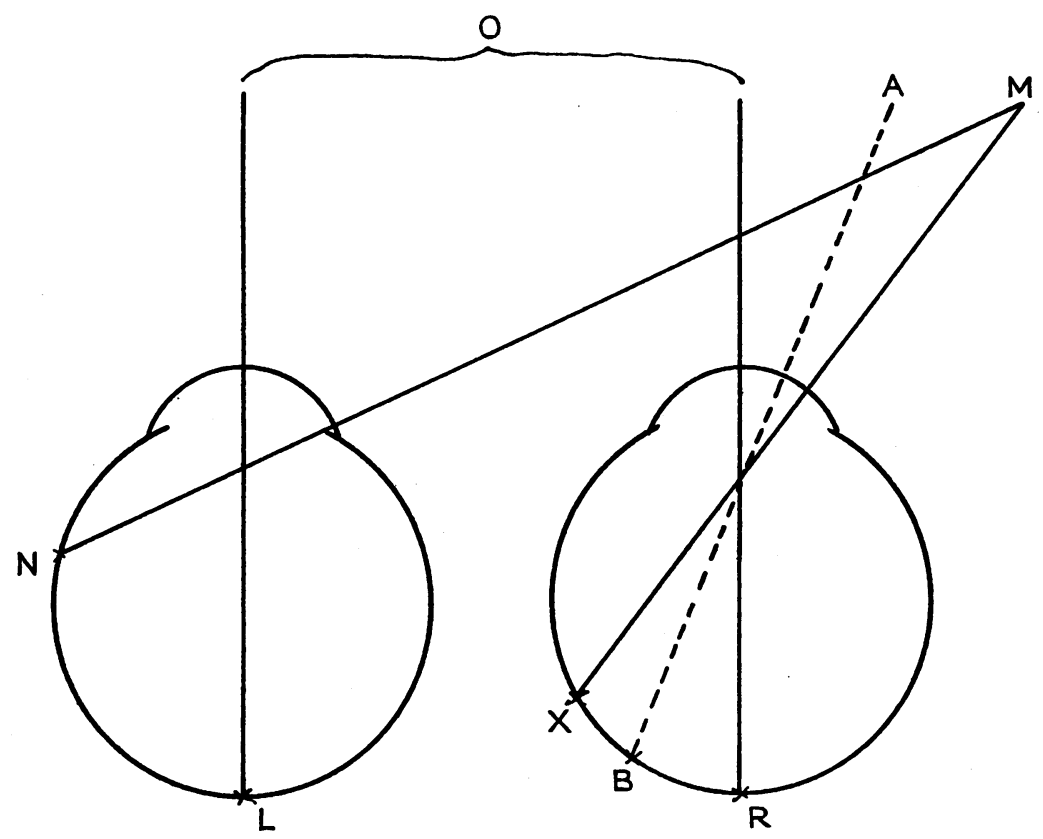

FIG. 4.

correspondence in which the left macula, while fixing, had the same local sign as the point $\mathrm{X}$ in the right retina.

Now, while L. fixes, the image of $M$ at $N$, in the fixing eye, will be the only image of $M$ seen in the binocular field, because its image at $\mathrm{X}$ will be suppressed. Therefore, $\mathrm{M}$ will be seen as if it were to the right of $\mathrm{O}$, and not superimposed on $\mathrm{O}$. Therefore some meridian $A B$, in the right part of the field will then assume the same directional value as the fixing visual line. Again the correspondence of the fixing visual line and the line AB will change-the point $B$ gradually approaching $R$. As this occurs, the suppression around $B$ will become less and less as the retinal 
visual acuity rises. When $B$ reaches $R$, and correspondence becomes normal, suppression will cease-or may be of the alternating macular type which is the manifestation of normal retinal rivalry.

\section{Summary}

1. In the normal binocular field there are two visual lines which meet upon the fixation object. This arrangement permits binocular fixation with little or no suppression.

2 . In squint there are two visual lines which do not meet upon the fixation object. This would cause diplopia and confusion unless suppression occurred.

3. In squint there are two areas of suppression in the binocular field.

4. There occurs : (a) Suppression of the diplopia image.

(b) Suppression of the part of the field which has the same local sign as the fixing macula. This suppression prevents confusion.

5. Suppression of the area described in $4(a)$ is not related to retinal correspondence.

6. Suppression of the area described in $4(b)$ is intimately associated with retinal correspondence. It is directly associated with the angle of anomaly, in that the larger the angle of anomaly, the greater the area of suppression.

7. It has been shown clinically that as abnormal correspondence changes after operation and the angle of anomaly lessens, so does the area of suppression become smaller. When the angle of anomaly becomes $\mathrm{O}$, and correspondence is normal, there is no suppression.

8. It is suggested that the first step in the development of abnormal correspondence is macular suppression. Macular suppression in one eye (or part of the binocular field) alters the power of discriminating direction in the deviated component of the binocular field. This allows the abnormal perception of direction to develop.

\section{BIBLIOGRAPHY}

1. Travers, T. a'B.-Brit. Jl. of Ophthal., Vol. XXII, p. 577, 1938.

2. VON KRIES, J.-Arch. f. Ophthal., Vol. XXIV, p. 117, 1878.

3. Lot $Z$ E.-Referred to in Schafer's Text-Book of Physiology, Vol. II, p. 1137. Young J. Pentland, 1900. 